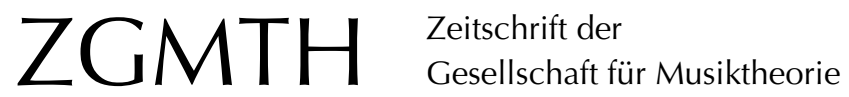

Arnecke, Jörn (2014): Im Spannungsfeld zwischen Handwerksregel und Klangvorstellung: Instrumentation als Hochschullehrgang. ZGMTH 11/1, 75-87.

https://doi.org/10.31751/793

(C) 2014 Jörn Arnecke

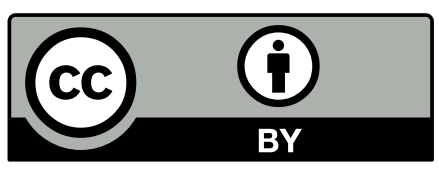

Dieser Text erscheint im Open Access und ist lizenziert unter einer Creative Commons Namensnennung 4.0 International Lizenz.

This is an open access article licensed under a Creative Commons Attribution 4.0 International License.

veröffentlicht / first published: 24/07/2015

zuletzt geändert / last updated: 19/02/2016 


\title{
Im Spannungsfeld zwischen Handwerksregel und Klangvorstellung: Instrumentation als Hochschullehrgang
}

\author{
Jörn Arnecke
}

ABSTRACT: Skizziert wird ein zweisemestriger Hochschulkurs im Fach >Instrumentation «. Lehrbeispiele zu Joseph Haydn und Paul Hindemith umreißen das Spannungsfeld zwischen Regelgebundenheit und klanglicher Freiheit. Hierbei werden Klavierauszüge instrumentiert und detailliert mit dem Original verglichen. Dieser stilgebundene Zugang zur Instrumentation vermittelt zunächst das Handwerk und fordert dann zu eigenständigen, kreativen Lösungen heraus.

The article outlines a two-term higher education course in sinstrumentation`. Examples from the works of Joseph Haydn and Paul Hindemith define a field of tension between technique and imagination. Own arrangement of vocal scores for instruments will be compared in-depth to the original. Such a style-oriented approach to instrumentation based on strong, time-dependent rules convey initially craftsmanship, and challenges subsequently a creative approach to sound color and timbre.

\section{Ist Instrumentation lehrbar?}

"Scoring for orchestra is thinking for orchestra. ${ }^{1}$

Für das Orchester zu denken - das ist eine Lehrpraxis und ein Anspruch, der sich im Gefolge des wichtigen Buches Thinking for orchestra von René Leibowitz und Jan Maguire $^{2}$ etabliert hat. Dahinter verbirgt sich ein Zusammenführen der technischen Aspekte des Instrumentierens bzw. Orchestrierens ${ }^{3}$ mit der Idee von »Instrumentation als kompositorisch integralem Faktor «. ${ }^{4}$ In diesem Spannungsfeld zwischen Regel und Freiheit, zwischen Wissensvermittlung und erwünschter Klangfantasie bewegen sich alle Instru-

$1 \quad$ Adler 2002, 547.

2 Leibowitz/Maguire 1960. Im deutschsprachigen Raum wird das Buch bislang wenig beachtet: Das ausführliche Literaturverzeichnis bei Jost 2004 etwa führt es nicht auf.

3 Ertugrul Sevsay unterscheidet zwischen den spieltechnischen Möglichkeiten der Instrumente (Instrumentation) einerseits und dem Zusammenwirken der Instrumente (Orchestration) andererseits (2005, 17f.).

4 Jost 2004, 12. 
mentationslehren. Hierbei lässt sich im groben historischen Überblick eine Akzentverschiebung ausmachen: Während Traktate aus dem 15. und 16. Jahrhundert vor allem oder ausschließlich einen Überblick über die Instrumente der Zeit gaben ${ }^{5}$, wurde im 20. Jahrhundert zunehmend die kompositorische Idee als Ausgangspunkt unterstrichen. Besonders markant vertreten Walter Gieseler, Luca Lombardi und Rolf-Dieter Weyer diesen Ansatz in ihrem Lehrwerk Instrumentation im 20. Jahrhundert ${ }^{6}$ :

Alle bis jetzt erschienenen Bücher über Instrumentation, Instrumentationslehre, Orchestration, Instrumentierung sind (seit dem 18. Jahrhundert) angesiedelt zwischen Instrumentenkunde und Instrumentation als kompositorischem Vorgang, zwischen Information über Einzelinstrumente und über ihr Zusammenwirken mit dem Ziel musikalischer Gestaltung. Es wäre zu zeigen, daß unser neues Buch tatsächlich zum ersten Mal versucht, das Problem der Instrumentation (im Vollsinne) nur von kompositorischen [...] Prinzipien aus anzugehen. ${ }^{?}$

Das Spannungsfeld zwischen Handwerk und Kreativität zeigt sich auch beim Versuch, die Frage zu beantworten, inwieweit Instrumentation lehrbar sei. Wenn Autoren die Lehrbarkeit grundsätzlich anzweifeln, folgen sie der Vorstellung, dass sich generell alle kreativen Prozesse einer didaktischen Vermittlung entziehen. Hector Berlioz schreibt 1844:

Von ihrer poetischen Seite betrachtet, läßt sich diese Kunst ebensowenig lehren, als die Kunst, schöne Akkordfolgen und originelle, kräftig-rhythmische Formen zu erfinden. ${ }^{8}$

Nikolai Rimsky-Korsakow formuliert ähnlich 1891:

To orchestrate is to create, and this is something which cannot be taught. ${ }^{9}$

Und Richard Strauss wird 1904 bei dieser Frage polemisch:

In der Instrumentationskunst, wie wohl in allen künstlerischen Dingen, ist es mit theoretischen Büchern eine mißliche Sache. Ich behaupte: ein mit Kompositionstalent begabter Musiker, der als Geiger oder Bläser in einem Orchester tätig ist, wird von vornherein ohne jede Kenntnis der Instrumentationslehre ein größeres Geschick für Orchestrationskunst besitzen, als der ebenfalls zum Tondichter berufene Pianist ,So und So oder der federgewandte Kritiker 'Nie und Nimmer, der zwar fleißig Instrumentationslehre studiert, aber nie Orchesterinstrumente näher zu Gesicht bekommen hat, als in einer Entfernung von der Stuhlreihe seines Platzes im Konzertsaal bis zum Konzertpodium. $^{10}$

5 Für eine detaillierte Übersicht vgl. Jost 2004, $147 \mathrm{ff}$.

6 Gieseler/Lombardi/Weyer 1985.

7 Ebd., 4.

8 Berlioz/Strauss 1904, 2.

9 Rimsky-Korsakow 1964, 2.

10 Berlioz/Strauss 1904, Vorwort (o.S.). 
Wie soll ein Hochschulkurs über Instrumentation die Aspekte Handwerk und Kreativität verbinden und gewichten? Dieser Beitrag versucht anhand von zwei Unterrichtsbeispielen aus unterschiedlichen Jahrhunderten eine Einordnung: Mit technischen Aspekten beschreibt er den Instrumentationsstil eines Komponisten und damit ein für diesen gültiges Regelwerk. Anhand von mehreren Lösungen, die innerhalb dieses Regelwerks möglich wären, untersucht er das Maß an Freiheit und Inspiration, das den Komponisten in seiner Zeit geleitet hat.

\section{Zur Konzeption eines Instrumentations-Kurses}

Die Beispiele folgen einer Konzeption, die der Autor für zweisemestrige Kurse ıInstrumentation sentwarf und an der Hochschule für Musik und Theater Hamburg sowie der Hochschule für Musik FRANZ LISZT Weimar umsetzte. Instrumentation wird dabei ausdrücklich als stilgebundene Instrumentation verstanden: Kursziel ist also nicht die ungebundene Stimulation von Klangfantasie, sondern der Nachvollzug einer historischen Entwicklung des Orchesters anhand von eng umrissenen Übungen, die von den Studierenden bearbeitet werden. Dies unterscheidet diesen Vorschlag etwa von Genre-übergreifenden Ansätzen wie bei Ulrich Kaiser und Carsten Gerlitz ${ }^{11}$ oder von systematischen Arbeiten wie bei Peter Jost. ${ }^{12}$

Der hier skizzierte Kurs beginnt mit Instrumentation bei Joseph Haydn und endet bei György Ligeti. Voraussetzung dabei ist, dass die Studierenden (aus den Fächern Komposition, Dirigieren oder Schulmusik) ein Grundwissen über Stil und Instrumentenkunde mitbringen. Wesentlich beeinflusst wurde die Konzeption durch ein einjähriges Studium am Pariser Conservatoire National Supérieur und den dortigen Kurs Orchestration beim französischen Komponisten Marc-André Dalbavie. Das erste der beiden folgenden Lehrbeispiele, ausführlich behandelt und auf Dalbavie zurückgehend, soll belegen, dass in der Klassik klare Regeln für das Instrumentieren herrschen. Das zweite ist ganz bewusst dem 20. Jahrhundert entnommen, um zu zeigen, wie Handwerksregeln mit neuerer Musik vertieft werden können.

11 Hier wird die Abkehr vom stilgebundenen Instrumentieren zur Lehrmethode erhoben: »Ein reizvoller Stilbruch ist es, einen Komponisten einer anderen Epoche, z. B. des Hochbarock (wie Johann Sebastian Bach), für eine Orchesterbesetzung des 19. Jahrhunderts zu bearbeiten « (Kaiser/Gerlitz ${ }^{2} 2009$, 69). Der pragmatische Zugang zeigt sich u. a. auch daran, dass bei der Behandlung der klassischen Instrumentation (ebd., 43ff.) die Viola zunächst aus lesetechnischen Gründen ausgelassen wird.

12 Seine umfangreiche Darstellung versteht sich ausdrücklich nicht als Lehrwerk: „Selbstverständlich soll der vorliegende Band kein Instrumentations-Lehrbuch sein, keine Anleitung und auch keine bloße Beispielsammlung. Vielmehr versucht er, den so vielfältigen und nur schwer übersehbaren Bereich unter einigen wesentlichen Gesichtspunkte in konzentrierter Weise aufzufächern « (Jost 2004, 9). 


\section{Grundprinzipien der klassischen Instrumentation bei Haydn}

Welche Vorstellung verbinden Studierende mit Instrumentation? In einer jüngst vom Autor durchgeführten Umfrage sollten Studierende des ersten Semesters einschätzen, wie groß die Bedeutung von `Technik/Handwerkı und `Fantasie/Inspiration` für die Befähigung zur Instrumentation ist. Möglich war jeweils eine Wertung von 1 (wird ivoll und ganzı zur Befähigung benötigt) bis 6 (ist zur Befähigung ıgar nicht` erforderlich). ${ }^{13}$ Das Ergebnis zeigt, dass beide Bereiche für wesentlich gehalten werden, 'Fantasie/Inspiration jedoch als bedeutsamer bewertet wird..$^{14}$ Derjenige Aspekt, der zweifellos lehrbar ist, wird damit für weniger wichtig gehalten.

Dies belegt: Unterricht im Fach Instrumentation kann einen Überraschungseffekt dadurch erzielen, dass er konkrete Regeln und Stilmerkmale nachweist und die Freiheit damit zunächst bewusst einschränkt (damit wäre ein wichtiger Unterschied zum offener gehaltenen Fach Arrangieren markiert). Erst die genaue Kenntnis von stilgebundenen Techniken der Instrumentation befähigt Studierende dazu, ihre Klangideen in einem stimmigen ästhetischen Rahmen zu entwickeln. Daher soll am Anfang auch immer für eine Standardbesetzung der jeweiligen Zeit instrumentiert werden. ${ }^{15}$

Die Musik Haydns eignet sich aufgrund ihrer Klarheit im Umgang mit dem Orchester ausgezeichnet, um erste wichtige Prinzipien und Regeln zu erlernen. Diese lassen sich so eindeutig formulieren, dass es für Aufgaben in bestimmten Bereichen nur eine einzige Lösung geben kann. Nach meiner Erfahrung im Unterricht ist es auch gerade am Anfang wichtig, Lösungen mit srichtig` und ‘falsch` und nicht nur mit sbesser zu bewerten. ${ }^{16}$ In anderen Bereichen jedoch darf und soll durchaus diskutiert werden, ob Instrumentationsentwürfe gleichrangig nebeneinander bestehen können oder ob einer dem anderen vorzuziehen ist.

13 Der Autor nahm die Umfrage am 22.10.2014 in seiner Übung Instrumentenkunde/Akustik an der Hochschule für Musik FRANZ LISZT Weimar vor. Es beteiligten sich 36 Studierende.

14 Der Mittelwert der Antworten betrug für den Aspekt `Technik/Handwerkı 2,2, für `Fantasie/Kreativität 1,9. Insgesamt ergibt sich bei `Fantasie/Kreativität eine Verschiebung in Richtung einer höheren Wichtigkeit: Für 1 (`voll und ganz`) votierten mit 38,9\% deutlich mehr als bei `Technik/Handwerkı (25,0\%); ähnlich fielen die Antworten bei 2 (38,9\% / 41,7\%) und 3 (19,4\% / 22,2\%) aus, im unteren Bereich entfielen mehr Wertungen auf sTechnik/Handwerkı (4: 8,3\% und 5: 2,8\% gegenüber 4: 2,8\% und 5: 0,0\% bei `Fantasie/Kreativität`). Für 6 (`gar nicht`) entschied sich bei beiden Aspekten niemand.

15 Die ästhetische Differenz einer stilfremden Besetzung kann nur dann erhellend sein, wenn sie als solche bewusst eingesetzt wird. Auch dies setzt jedoch voraus, zunächst ein stilgebundenes Regelwerk zu lernen, bevor die Fantasie eigene Wege gehen darf.

16 Hierbei würde ich den Akzent etwas anders setzen als Sevsay. Er schreibt in seinem hervorragenden Lehrbuch unter der Überschrift »Richtig und falsch«" (Sevsay 2005, 284): »Die Instrumentation des lernenden Arrangeurs kann sich mehr oder weniger stark von der originalen unterscheiden, und das ist völlig in Ordnung. Solange der Student im Rahmen des Stils des jeweiligen Komponisten oder der Epoche bleibt, eine planvolle und solide Arbeitsweise an den Tag legt und keine technischen Probleme verursacht [...], ist seine Instrumentation eine erfolgreiche. Der begleitende Lehrer wird sogar oft überrascht sein, welch schöne Alternativen zu den originalen Partituren von den Studenten erarbeitet werden.« Ein Lehrbeispiel für eine Einführungsstunde sollte jedoch aus meiner Sicht den Spielraum stilistisch möglicher Entscheidungen gering halten. Aus den Kategorien ^richtig^ und `falsch k kann ein Regelwerk erwachsen, das die Vorstellung von Instrumentation als Ausdruck von Klangfantasie relativiert. 
In einer ersten Stunde sollen folgende Grundlagen erarbeitet werden: Aufbau des klassischen Sinfonieorchesters, Technik der Kontrastbildung, Denken der Klassik in Orchestergruppen, Einblick in die Registerlagen der Bläser. Das Beispiel entstammt dem 1. Satz aus Haydns Sinfonie Nr. 97, C-Dur ${ }^{17}$ (siehe Bsp. 1). Die Aufgabe besteht aus drei Teilen $^{18}$ : der Instrumentation von knapp drei Takten Orchestertutti im Unisono sowie zweimal dem Wechsel zwischen Forte und Piano im Orchestersatz. Die Besetzung ist gegeben: Sie umfasst je zwei Flöten, Oboen, Fagotte; je zwei Hörner und Trompeten in C; Pauke; Streicher.

Arbeitsauftrag:

Instrumentieren Sie die markierten Stellen des ersten Satzes der Sinfonie Nr. 97 von Joseph Haydn für folgende Besetzung: $2 \mathrm{Fl}-2 \mathrm{Ob}-2 \mathrm{Fg} ; 2 \mathrm{Hr}(\mathrm{C})-2 \mathrm{Trp}$ (C); Pk c-G; Streicher
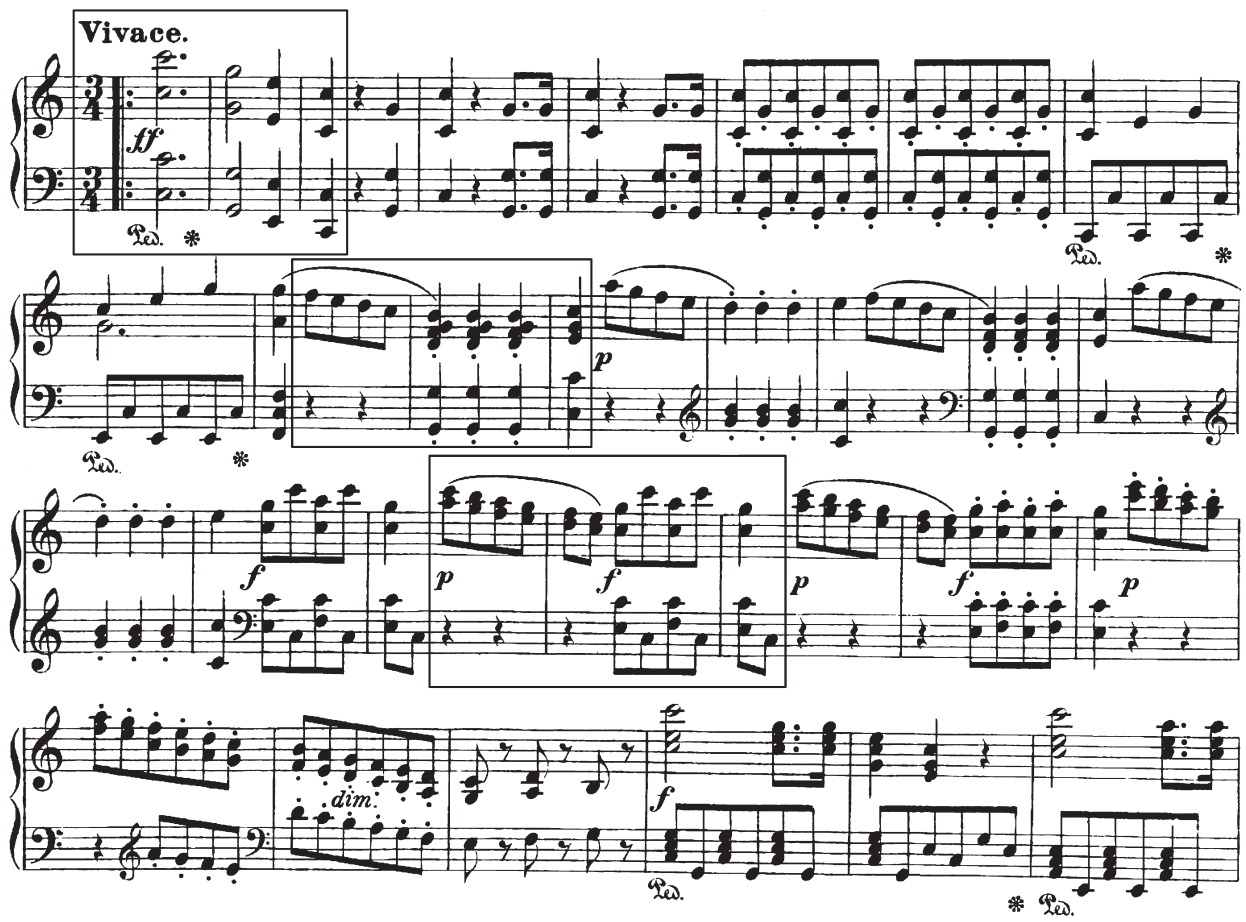

Beispiel 1: Aufgabe zu Joseph Haydn, Sinfonie Nr. 97, 1. Satz, Fassung für Klavier T. 14-42 ${ }^{19}$

17 Die Wahl eines Satzes in C-Dur ist eine bewusste didaktische Entscheidung, um das Problem der Transposition (abgesehen von Oktavtranspositionen) zunächst ausklammern zu können. Mit diesem Beispiel folge ich dem Unterrichtsbeginn, wie ihn Marc-André Dalbavie in Paris praktizierte.

18 Diese Aufspaltung in Teilaufgaben, die der Autor vornahm, soll einerseits Zeit und Schreibarbeit ersparen. Andererseits wird damit von Beginn an geschult, dass eine Entscheidung für eine Instrumentations-Konstellation nicht nur punktuell getroffen werden darf, sondern immer mit Blick auf einen größeren Abschnitt erfolgen muss. Die Verbindung zwischen den Teilaufgaben muss strukturell mitbedacht werden. 


\section{Entscheidungen und Regeln}

Das Problem des ersten Aufgabenteils lässt sich nun auf den schlichten Nenner bringen: Wie instrumentiert man in der Klassik einen absteigenden C-Dur-Dreiklang im Tutti? Dies scheint auf den ersten Blick sehr einfach zu sein - bei der konkreten Ausarbeitung stellen sich jedoch folgende Probleme, aus denen man durch den Vergleich mit Haydns Partitur (siehe Bsp. 2) wesentliche klassische Regeln ableiten kann:

\section{Problem 1: Werden die doppelten Bläser im Unisono oder in Oktaven gesetzt?}

Lösung: Die Fortissimo-Angabe ${ }^{20}$ weist darauf hin, dass ein scharfer, konturierter Gesamtklang angestrebt ist. Alle Instrumente sollen in ihrer besten, lautesten Lage spielen. Daher ist der Unisono-Klang vorzuschreiben: Oktaven würden den Klang unnötig binden und die jeweils zweiten Bläser in ungünstige Lagen führen.

\section{Problem 2: Welche Lagen sind für die Holzbläser zu wählen?}

Lösung: Für die Flöten ist es klar: $c^{3}$ bis $c^{2}$. Bei den Oboen stellt es sich schwieriger dar: Ist $c^{3}$ als Fortissimo-Beginn noch erlaubt oder schon zu hoch? $d^{3}$ kann für die Oboen als oberer Grenzton in der Klassik ${ }^{21}$ gelten, $c^{3}$ ist also noch erlaubt, ja wegen des scharfen Klanges sogar erwünscht. Sollen die Fagotte von $c^{1}$ bis $c$ spielen oder von $c$ bis $C$ ? Der Ton $C$ würde einen zu dicken Klang erzeugen, das klassische Klangideal ist hingegen hoch und hell; richtig ist also $c^{1}$ bis $c$.

\section{Problem 3: Welche Lagen sind für die Blechbläser zu wählen?}

Vorweg sollte daran erinnert werden, dass es sich bei Hörnern und Trompeten selbstverständlich um Naturinstrumente handelt, die also auf die Töne der Obertonreihe begrenzt sind. Die C-Trompeten klingen wie notiert, die C-Hörner eine Oktave tiefer als angegeben.

Lösung: Das Dreiklangsmotiv des Themas korreliert damit, dass Hörner und Trompeten daran in Dur uneingeschränkt mitwirken können - dies beweist schlagend, wie Themenerfindung und Instrumentation miteinander zusammenhängen. Ein ähnliches Thema in c-Moll wäre in der sinfonischen Musik nicht denkbar, da die Blechbläser die Terz nicht mitspielen könnten. In der Klaviermusik jedoch wird ein solches Thema sehr wohl komponiert, wie etwa der erste Satz von Wolfgang Amadé Mozarts c-Moll-Sonate KV 457 belegt.

19 Der vorliegende Ausschnitt ist einer Fassung für Klavier von August Horn (Leipzig: Peters o. J.) entnommen.

20 Man beachte in Beispiel 2 die Angabe Forte (und nicht Fortissimo wie im Klavierauszug) in den Bläsern und in der Pauke. Für die Diskussion der Aufgabe ist es aber, wie sich gezeigt hat, fruchtbar, das Fortissimo im Klavierauszug (das aus den Violinen übernommen wurde) beizubehalten.

21 Vgl. Joseph Haydn: Sinfonie Nr. 97, 1. Satz, Takt 53 ( $d^{3}$ in der 1. Oboe) und Takt 108 (die Oboen spielen nicht $e^{3}$, sondern eine Oktave tiefer). 
Doch kommen wir zurück zu der Frage der Lagen. Für die Trompeten ist es eindeutig: $c^{3}$ wäre zu hoch, also bleibt nur $c^{2}$ bis $c^{1}$. Kompliziert sind die Hörner: $c^{2}$ (klingend) wäre zu hoch, c aber als Lage zu matt; die strahlendsten Horntöne würden so für den Fortissimo-Beginn verloren gehen. Haydn wählt deshalb eine Fassung entgegen der Bewegungsrichtung des Themas. Die Hörner beginnen auf $c^{1}$ (klingend), schwingen sich dann zum $g^{1}$ auf und steigen erst danach ab. Dies wird im Unterricht als Überraschung registriert werden. Hier eröffnet sich bereits das Spannungsfeld zwischen objektivierbarem Regelwerk und individueller Entscheidung: Alternativlos ist Haydns Lösung nicht - aber sie hat unbestreitbare Qualität. (Anhand einer Aufnahme kann demonstriert werden, dass die Aufwärtsbewegung der Hörner nicht den Gesamteindruck einer Abwärtsbewegung stört.)

\section{Problem 4: Was macht die Pauke auf dem Ton e?}

Lösung: Die Pauke kann das Unisono nur auf den Tönen $c$ und $G$ unterstützen, die ihr zur Verfügung stehen. Für einen fehlenden thematischen Ton gibt es in der Klassik für die Pauke zwei Möglichkeiten: Entweder der Rhythmus wird mitvollzogen unter Verzicht auf die exakte Tonhöhe (wie z. B. beim Kopfmotiv aus Haydns Sinfonie Nr. 5322), oder der Ton wird einfach weggelassen. Hierfür entscheidet sich Haydn bei diesem Beispiel, er kürzt darüber hinaus die Töne auf Viertelnoten, um die Akzentuierung der Taktschwerpunkte zu unterstreichen.

\section{Problem 5: Welche Lagen sind für die Streicher zu wählen?}

Lösung: Für Violoncello und Kontrabass gilt zunächst das gleiche Argument wie für die Fagotte, ein gewichtiges anderes tritt noch hinzu: Die Kontrabässe könnten das $C_{1}$ gar nicht spielen (der Tonumfang von Viersaitern reicht ja nur bis $E_{1}$ ), sie müssten die Linie abbiegen, was unschön wäre und die Einheit von Violoncello und Kontrabass verletzen würde. Die Violen klingen in der Lage $c^{2}$ bis $c^{1}$ strahlender als eine Oktave tiefer, diese Lage ist also zu bevorzugen, ebenso für die 2. Violinen. Die 1. Violinen wären auch von $C^{3}$ bis $c^{2}$ denkbar, in Oktavspannung zu den 2. Violinen, im Unisono mit Flöten und Oboen; Haydn setzt sie jedoch von $c^{2}$ bis $c^{1}$, im Unisono mit den 2. Violinen und den Violen, was den hohen Bläsern die Klangkrone überlässt. Auch aus dem Streichersatz lassen sich daher Diskussionen über Entscheidungen, die der Komponist zu treffen hat, ableiten.

Die zweite und dritte Teilaufgabe behandeln den Kontrast. Sie sollen hier nur kurz dargestellt werden. Demonstrieren lässt sich an ihnen das Denken der Klassiker in Orchestergruppen. Die Piano-Antwort (T. 25ff.) wird dementsprechend nur von einer Gruppe (Holzbläser oder Streicher) gespielt; die Holzbläser ermöglichen dabei den größeren Kontrast (weniger Instrumente, der Klang wandert zudem nach hinten im Orchesterraum). Die Oberstimme legt Haydn dabei zunächst in die 1. Oboe - nicht in die 1. Flöte, da ein Instrument gefordert ist, das genügend Präsenz mitbringt, um im Nachhall der

22 Joseph Haydn, Sinfonie Nr. 53, 1. Satz, Takt 1. 


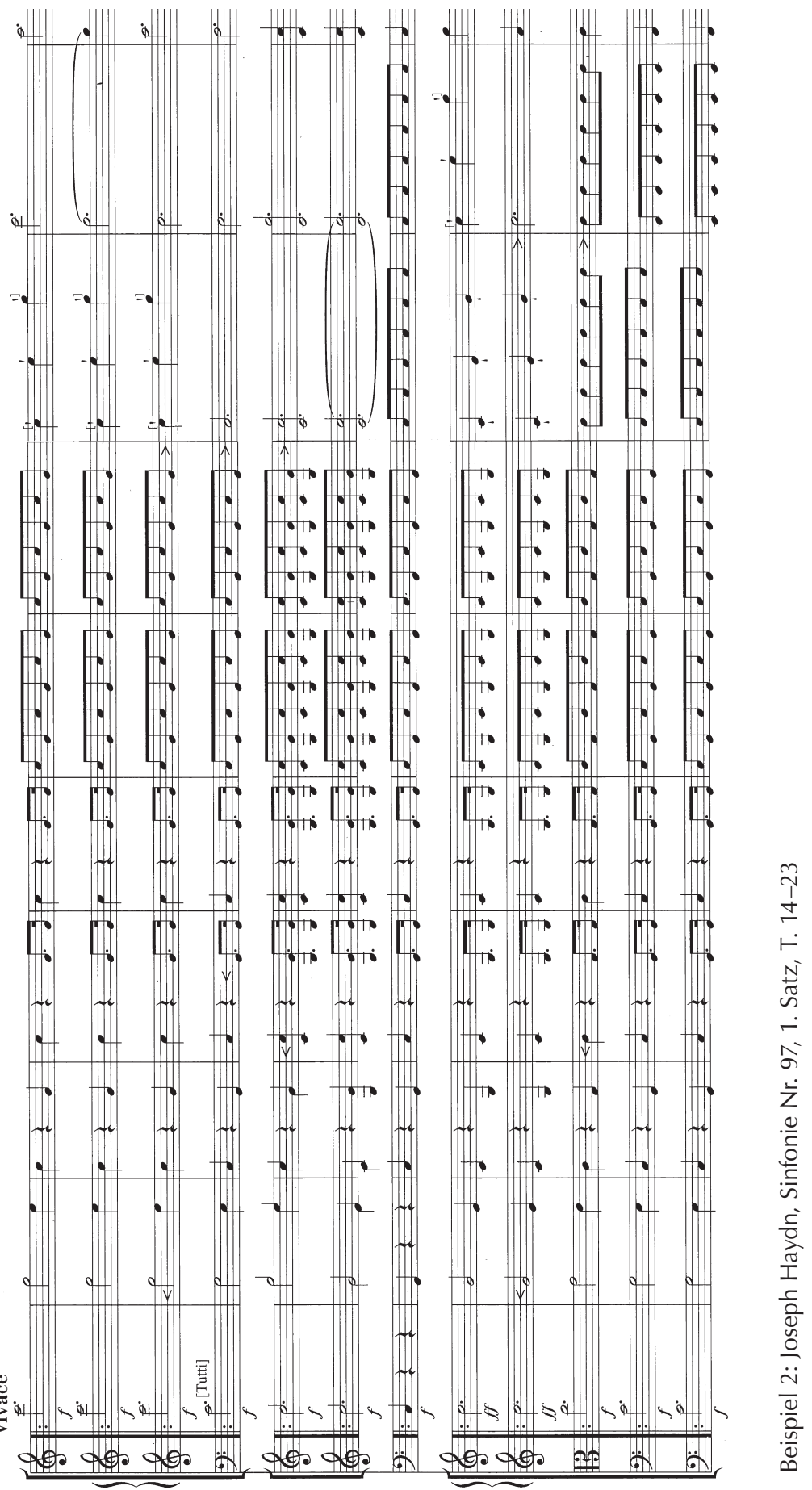


Fortissimo-Akkorde ${ }^{23}$ leise hervorzutreten. Sogar bei der Folgestelle (T. 31 ff.) lässt Haydn im Piano die Oboen spielen und nicht die Flöten, deren Einsatz in dieser Lage nach dem Tutti-Forte zu schwach wäre (dritte Teilaufgabe).

Anhand dieser Beispiele lässt sich deutlich zeigen, dass die klassische Instrumentation relativ wenig Spielraum für kreative Entscheidungen lässt. Instrumentation bei Haydn folgt einem klar strukturierten Orchesteraufbau mit eng umrissenen Aufgaben für die Orchestergruppen.

\section{Weiterführung im 20. Jahrhundert am Beispiel Paul Hindemiths}

Der Instrumentationskurs erarbeitet anschließend chronologisch Techniken aus Klassik und Romantik. Stichwortartig seien hier die Komponisten Mozart, Beethoven, Berlioz, Wagner, Bruckner, Strauss und Mahler genannt. ${ }^{24}$ Auch Instrumentation im 20. Jahrhundert kann mit einer ähnlichen Methodik erfasst werden. In der Aufgabe zu Paul Hindemiths Sinfonie Mathis der Maler ist der Anfang des Werkes im Klavierauszug ${ }^{25}$ notiert (Beispiel 3). Die >deutscher Technik Hindemiths schließt sich im Kurs an den französischen Orchestersatz des Impressionismus an. Der Gegensatz wird deutlich herausgearbeitet: Hindemith instrumentiert klar strukturiert, nicht auf ein Verwischen, sondern auf ein Schärfen der Konturen ausgerichtet.

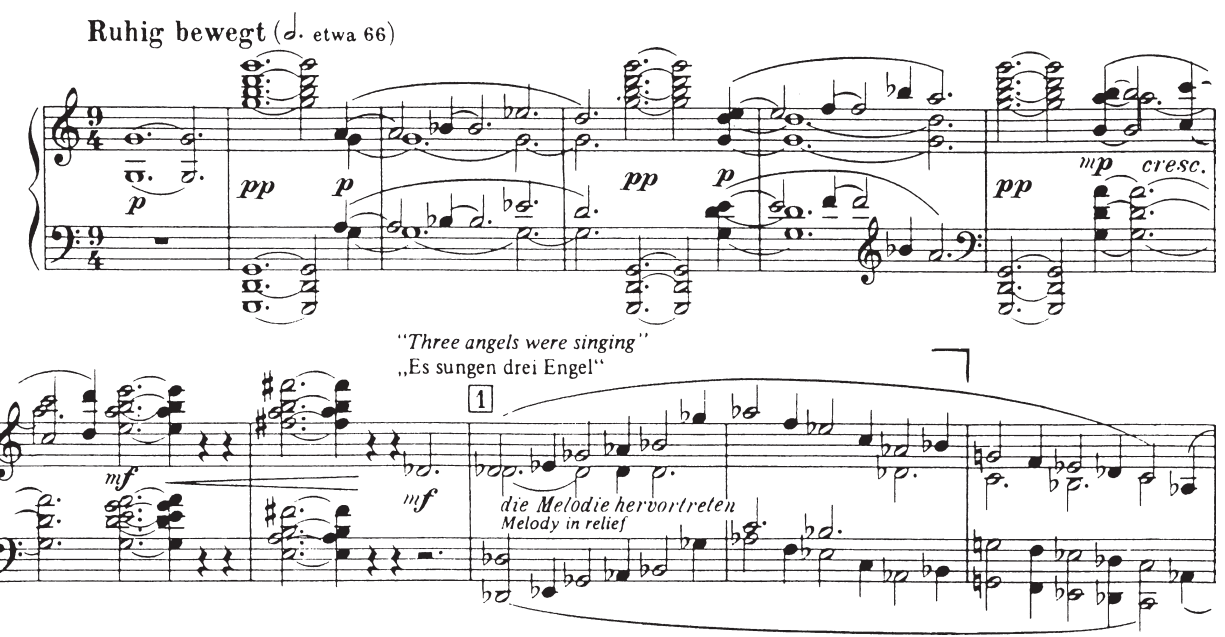

Beispiel 3: Paul Hindemith, Sinfonie Mathis der Maler, 1. Satz (Klavierauszug des Komponisten), T. $1-11$

23 Vgl. Anmerkung 20.

24 Aus Platzgründen kann dies hier nicht ausführlicher dargestellt werden.

25 Dieser erste Satz der Sinfonie eröffnet auch Hindemiths gleichnamige Oper, zu der er selbst den Klavierauszug anfertigte (Mainz: Schott 1935). 


\section{Engelkonzert}
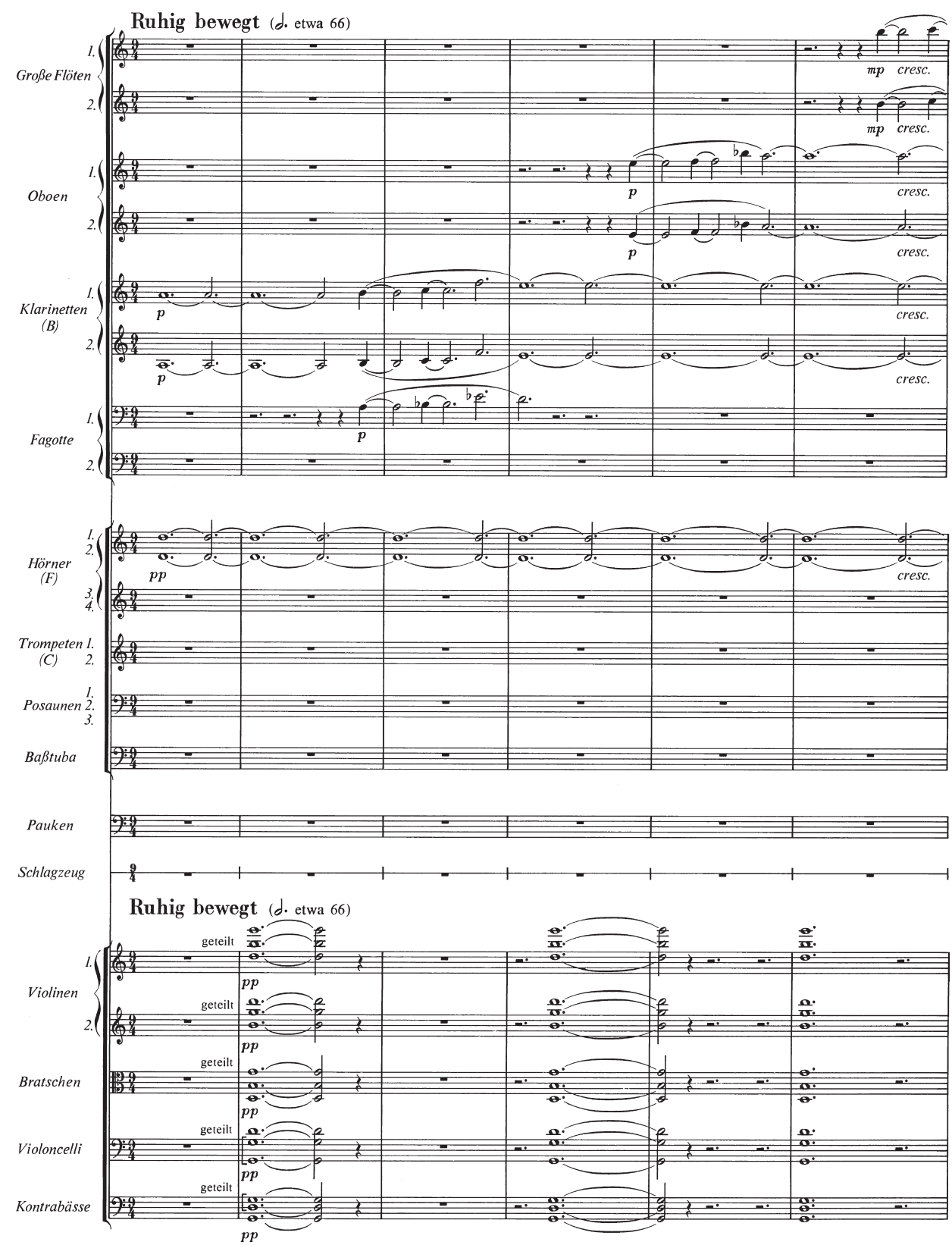

Beispiel 4: Paul Hindemith, Sinfonie Mathis der Maler, 1. Satz, T. 1-10 
Verschiedene Ereignisse werden durch verschiedene Gruppen voneinander abgehoben (Bsp. 4): So liegt die Ansatzoktave $g^{1}-g$ in den Bläsern (Hörner und Klarinetten), der darauf folgende, weit gespannte G-Dur-Akkord in den Streichern. Für die Sequenz des Eingangsmotives ergibt sich die logische Folge: Klarinetten, Oboen, Flöten, vom matten über den schärferen zum leuchtenden Klang, immer in Oktaven geführt, um eine hohe Homogenität zu erhalten. Eine Überraschung liegt darin, dass Hindemith die Klarinetten als einziges Holzbläserpaar in ihrer Oktavlinie unterstützt - nämlich mit einem Fagott. Dies zeigt wieder eine individuelle Entscheidung jenseits einer starren Systematik an: Die erste Oktavlinie soll als Wandlung aus dem Anfangsklang hervorgehen, durch eine leicht abdunkelnde Farbe unterstützt werden. Für das Choralmotiv »Es sungen drei Engel« (T. 9 m. A.) bietet sich die Lösung an, die auch Schubert für das dritte Thema seiner Großen C-Dur-Sinfonie ${ }^{26}$ wählt: drei Posaunen im Unisono. Hier wären auch andere Klangfarben denkbar, etwa die Hörner - was dann wiederum Auswirkungen auf die Instrumentation vorher hätte: Denn ein Reiz der Lösung Hindemiths ist, dass die Posaunen als Klangfarbe mit Einsetzen des Chorals neu hinzutreten.

Die Verteilung der verschiedenen Ereignisse auf verschiedene Gruppen lässt sich aus Hindemiths Personalstil und seiner Ästhetik ableiten und begründen. Aber wäre die Verteilung nicht auch umgekehrt denkbar: der Akkord in den Bläsern, das Eingangsmotiv sequenziert in Viola, Violine II und Violine I? Wenn man diese Möglichkeit durchspielt, ergibt sich eine ebenfalls zwingende Lösung, allerdings wird der Anfangsklang nicht ganz so differenziert ausfallen können wie im Streicherapparat. Gerade in der Tiefe fehlt es an Instrumenten $\left(G_{1}\right.$ ist ohne Kontrafagott mit Holzbläsern nicht spielbar), insbesondere solchen, die in dieser Lage noch ein Pianissimo spielen können. Dass ein Austausch der Gruppen jedoch prinzipiell denkbar ist, zeigt Hindemith später im Stück (T. 34-37): Hier legt er tatsächlich die Oktavlinien in die Streicher, den Akkord in die Holzbläser und Hörner (hier Des-Dur, deshalb ist der tiefste Ton des Akkordes, Des, durch das zweite Fagott realisierbar).

\section{Handwerk oder Inspiration?}

Der Schwerpunkt dieser Aufgabe liegt immer noch auf Instrumentation als Handwerk. Klangliche Inspiration kommt bei Entscheidungen über die Klangfarbe hinzu. Die Lösungen werden weitaus reichhaltiger ausfallen, die Diskussionen über verschiedene Optionen mehr Raum einnehmen. Die Freiheit zu individuellen Entscheidungen erweist sich aber immer noch eng gekoppelt an ein bestimmtes stilistisches Regelwerk.

Für die zweite Hälfte des 20. Jahrhunderts lassen sich reizvolle Aufgaben stellen, die einen ganz anderen Zugang verlangen, nämlich in der von Gieseler/Lombardi/Weyer beschriebenen Weise eine Klangkonzeption zu erstellen, welche die gesamte Besetzung in den Blick nimmt und nicht von einem Klaviersatz ausgeht - etwa die Aufgabe, einen gegebenen Cluster $a^{2}-c^{3} z u$ instrumentieren und mit Flageolett-Tönen der Streicher zu umhüllen. In Ligetis Atmosphères kann man eine hochdifferenzierte Orchesterpassage ${ }^{27}$ finden, der die gleiche kompositorische Aufgabe zugrunde liegt.

26 Franz Schubert, Sinfonie D 944, 1. Satz, Takte $199 \mathrm{ff}$.

27 György Ligeti, Atmosphères, Takte 88ff. 


\section{Schulung der Klangfantasie durch Instrumentation von Klavierstücken}

In jedem Semester sollen die Studierenden mindestens eine größere zusammenhängende Instrumentation erarbeiten - in der Regel mit einem Klavierstück als Vorlage. Ging es bei den methodisch zugespitzten Aufgaben um das Erlernen eines stilistisch gebundenen Handwerks, so sollen nun eigene, begründete Klangentscheidungen getroffen werden. Denn in der Übertragung eines Werkes für Klavier auf das Orchester ist ein individuelleres Vorgehen nötig - etwa in Bezug auf den verwendeten Tonraum, auf das Übersetzen spezieller Klaviersatz-Techniken oder das Hinzufügen von Pedaltönen. Wesentlich ist aber auch hier ein methodisch klares Vorgehen: Der Überblick über die strukturellen Ereignisse muss am Anfang stehen. Um dies zu veranschaulichen, eignet sich z. B. in einem zweiten Semester Béla Bartóks Stück Obertöne aus Mikrokosmos, Band 4 hervorragend, da es sich in wenige Kategorien einteilen lässt (Bsp. 5): Anschlag, Nachklang, Legato-Linien, Vor- und Nachbereitung der Anschläge. Durch die besondere Rolle des Nachklangs mit den stumm gedrückten Tasten in der linken Hand wird außerdem das Augenmerk auf das Orchesterpedal gelegt. Eine solche Instrumentation stellt eine klingende Analyse dar, und damit treten die Verwandtschaftslinien zwischen beiden Fächern deutlich hervor: Was in der Analyse an Strukturen aufgedeckt wird, muss durch die Instrumentation klanglich umgesetzt werden.

\section{Allegro non troppo, un poco rubato, $d=c 2110$}

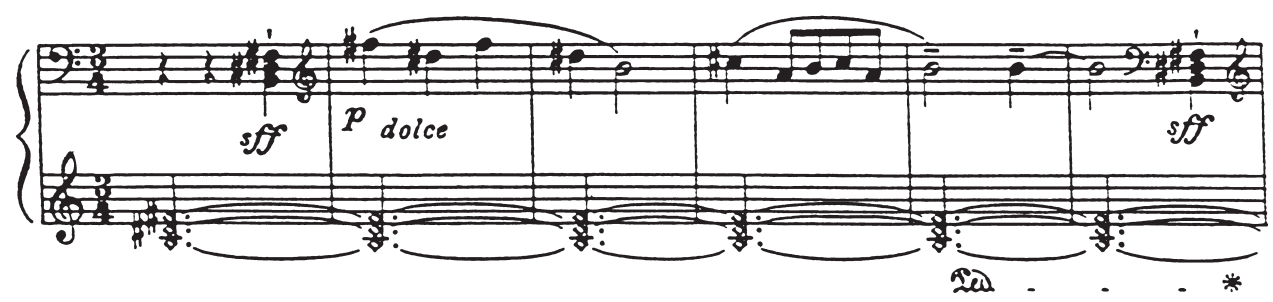

Beispiel 5: Béla Bartók, Obertöne aus Mikrokosmos, Bd. 4, T. 1-6

Wichtig als methodische Alternative im Unterricht ist zudem die Verwendung einer Aufnahme: Eine Instrumentationsanalyse kann, wenn ein Stil vertraut geworden ist, auch als Höranalyse erfolgen. Unverzichtbar bleibt gleichwohl das Lesen, Studieren, Analysieren und Präsentieren von Partituren verschiedener Epochen. Und ein gemeinsamer Konzertoder Probenbesuch ist nicht nur ein soziales Ereignis, sondern auch ein künstlerisches Erlebnis - und dies noch intensiver, wenn das Orchesterstück vorher im Kurs ausführlich behandelt wurde. ${ }^{28}$

28 Diese Teilbereiche des Instrumentationsunterrichts, die ich bereits bei einem Vortrag auf dem GMTH-Kongress 2004 in Köln vorstellte, finden sich in ähnlicher Weise auch bei Sevsay 2005. Er bevorzugt für seine Aufgaben gegenüber dem Klavierauszug das Particell, insbesondere um den Einsatz der Holzbläser lenken zu können. 


\section{Fazit und Ausblick}

Wie hier anhand von Lehrbeispielen aus zwei verschiedenen Jahrhunderten gezeigt wurde, positioniert sich stilgebundene Instrumentation im Spannungsfeld zwischen Handwerk und Klangvorstellung: Ausgehend von bestimmten stilistisch-ästhetischen Regeln fordern die gewählten Beispiele zunehmend die klangliche Kreativität für individuelle Lösungen heraus. Dieses Vorgehen vermag auch Studierende, die sich in Fragen der Instrumentation nicht für übermäßig inspiriert halten, in das Fach einzuführen und ihnen zu einem versierten Umgang mit dem Orchester zu verhelfen. Die Fantasie derjenigen Studierenden, die über viele Klangideen verfügen, wird möglicherweise durch Regeln zunächst gezügelt. Sie können später jedoch ihre Kreativität viel bewusster und zielgerichteter einsetzen.

Zudem vermag Instrumentation in dieser Weise wichtige Bezüge zu anderen Fächern innerhalb der Hochschulbildung herzustellen: Sie vermittelt Analyseeinsichten, die sich nur erschließen, wenn man den Denkprozess eines Komponisten für das Orchester aktiv nachvollzieht. Sie liefert Erkenntnisse, wie Themenbildung und formale Gestaltung durch Instrumentationsfragen ausgelöst und beeinflusst werden und wie sich dadurch die Anlage einer Sinfonie von der etwa einer Klaviersonate unterscheidet. Sie verbessert weiterhin das stilistische Feingefühl für den Klang und die Klangdisposition einer Epoche. Und die Studierenden im Fach Komposition verfeinern ihre Klangvorstellung und erhalten Handwerkszeug im Umgang mit dem Orchester, ja mit größeren Besetzungen ganz allgemein.

\section{Literaturverzeichnis}

Adler, Samuel (2002), The Study of Orchestration, 3. Auflage, New York: Norton.

Berlioz, Hector / Richard Strauss (1904), Instrumentationslehre, Leipzig: Peters.

Gieseler, Walter / Luca Lombardi / Wolf-Dieter Weyer (1985), Instrumentation in der Musik des 20. Jahrhunderts, Celle: Moeck.

Jost, Peter (2004), Instrumentation. Geschichte und Wandel des Orchesterklangs, Kassel u. a.: Bärenreiter.

Kaiser, Ulrich / Carsten Gerlitz, (2005), Arrangieren und Instrumentieren. Barock bis Pop, Kassel u. a.: Bärenreiter.

Leibowitz, René / Jan Maguire (1960), Thinking for Orchestra, New York: Schirmer. Rimsky-Korsakow, Nikolai (1964), Principles of Orchestration, New York: Dover.

Sevsay, Ertugrul (2005), Handbuch der Instrumentationspraxis, Kassel u. a.: Bärenreiter. 